\title{
Endothélines et leurs récepteurs: de nouveaux acteurs du développement
}

La maladie de Hirschsprung ( $\mathrm{MH}$ ) est une malformation digestive fréquente puisqu'elle touche un enfant sur 5000 naissances [1]. Dans sa forme classique, elle se traduit par une occlusion néonatale sévère, de traitement chirurgical. Le diagnostic repose sur l'étude histologique qui montre l'absence des cellules ganglionnaires assurant l'innervation intrinsèque de l'intestin terminal. Ces cellules ont pour origine les crêtes neurales (somites 1 à 7), ce qui définit la $\mathrm{MH}$ comme une neurocristopathie [2]. Si dans $80 \%$ des cas la $\mathrm{MH}$ est sporadique, l'existence de formes familiales est un argument majeur en faveur du rôle de facteurs génétiques. Ainsi, les analyses de ségrégations dans de grandes séries suggéraient de longue date un modèle multifactoriel duquel se dégageaient un ou plusieurs gènes autosomiques de faible pénétrance [3]. S'appuyant sur des cas familiaux, un premier gène de la $\mathrm{MH}$ a été localisé sur le bras long du chromosome 10 puis identifié [4-6] : il s'agit du protooncogène RET qui code pour un récepteur tyrosine kinase membranaire exprimé dans les cellules originaires des crêtes neurales $[7,8]$. Des mutations variables de RET sont associées à la $\mathrm{MH}$ dans des cas familiaux, mais également dans des cas sporadiques [9]. Si la faible pénétrance, l'expression variable des mutations de $R E T$ dans les formes familiales, et l'absence de corrélations génotypephénotype sont en faveur de l'existence de gènes modificateurs, de nombreux arguments plaidaient encore dans le sens d'une hétérogéneité génétique:

(1) La MH s'associe parfois à déterminées : anomalies chromosomiques (microdélétions du chromosome 13 , trisomie 21 ), ou autres neurocristopathies comme le syndrome de Waardenburg.

(2) Plusieurs mutants de mégacôlon aganglionique, souris lethal spotting, piebald lethal, et dominant spotting, se comportent chez la souris comme des caractères monofactoriels. Aucun de ces loci n'est allélique du locus RET murin, et, de surcroît, ces mutants présentent aussi des anomalies de coloration du pelage, ce qui n'est pas sans rappeler l'association chez l'homme de la $\mathrm{MH}$ au syndrome de Waardenburg $\left(\mathrm{m} / \mathrm{s} n^{\circ} 4\right.$, vol. 8 , p. $393 ; n^{\circ} 1$, vol. 11, p. 133).

(3) Enfin, dans une série de 80 malades, nous n'avons identifié une mutation de RET que dans $50 \%$ des cas familiaux et à peine $30 \%$ des cas sporadiques [10]. Bien que ce faible pourcentage puisse s'expliquer en partie par une insuffisance des techniques de détection de mutations [11], nous avons apporté la démonstration que, dans certains cas, des familles de $\mathrm{MH}$ sont exclues du locus RET [12].

S'appuyant sur un exceptionnel pedigree mennonite dans lequel de nombreux enfants sont atteints de $\mathrm{MH}$ ainsi que d'anomalies pigmentaires et sensorielles évoquant le syndrome de Waardenburg (mèche blanche frontale, hétérochromie des iris, surdité), le groupe de Chakravarti a cartographié un deuxième locus de susceptibilité à la $\mathrm{MH}$ sur le chromosome 13 , en position-13q22, entre les loci D13S162 et D13S170 [13]. Pour cela, ils ont tiré partie de la très forte consanguinité de ces enfants en combinant cartographie génétique par identité dans la des- cendance et recherche de déséquilibre de liaison. Ce travail les a conduits à identifier une mutation ponctuelle du gène codant pour le récepteur béta aux endothélines (EDNRB), un gène candidat par sa position [14]. Le gène $E D N R B$ code pour l'un des deux récepteurs des endothélines connus chez l'homme [15]. Le récepteur béta a une égale affinité pour les trois formes d'endothéline (ET1, 2 et 3) alors que le récepteur alpha a une affinité très prononcée pour l'endothéline de type 1. Ces récepteurs appartiennent à la superfamille des récepteurs de surface à sept domaines transmembranaires, couplés aux protéines $G$ [16]. Lors de la stimulation par leur ligand, EDNRA et EDNRB activent plusieurs signaux intracellulaires dont la voie de la phospholipase $\mathrm{C}$ qui conduit à l'accumulation cytoplasmique de calcium.

La mutation ponctuelle du gène $E D N R B$ mise en évidence chez les patients de cette grande famille américaine originaire du centre de l'Europe est une transversion $\mathrm{G} \rightarrow \mathrm{T}$ dans l'exon 4 conduisant au fauxsens tryptophane $\rightarrow$ cystéine au codon $276\left(\mathrm{~W}^{276} \rightarrow \mathrm{C}\right)$. Cette mutation se trouve au voisinage de l'extrémité extracellulaire du cinquième domaine transmembranaire du récepteur. Elle touche une séquence d'acides aminés tryptophane/phénylalanine qui présente une parfaite conservation évolutive dans tous les récepteurs des endothélines des vertébrés, y compris le récepteur alpha humain. Des mutations germinales ou somatiques d'autres récepteurs couplés aux protéines $G$ ont été mises en évidence dans un certain nombre de maladies génétiques 
Tableau I

UN GÈNE: PLUSIEURS MALADIES, UNE MALADIE : PLUSIEURS GÈNES

\begin{tabular}{|c|c|c|c|c|c|c|c|}
\hline Hérédité & $\begin{array}{c}\text { HOMME } \\
\text { Phénotype }\end{array}$ & Chromosome & Gènes & Chromosome & $\begin{array}{c}\text { Sout } \\
\text { Mutant spontané }\end{array}$ & ${ }_{\text {Knock-out }}^{\text {RIS }}$ & Hérédité \\
\hline$A D$ & $\begin{array}{c}\text { MH, } \\
\text { MEN2AMEN2B } \\
\text { FMTC }\end{array}$ & $10 q 11.2$ & RET & 6 & - & $\begin{array}{c}\mathrm{MH} \\
\text { Agénésie rénale }\end{array}$ & AR \\
\hline AR & $\begin{array}{c}\text { MH, } \\
\text { anomalies } \\
\text { pigmentaires, } \\
\text { surdité }\end{array}$ & $13 q 22$ & EDNRB & 14 & $\begin{array}{c}\text { Piebald lethal }(s) \text { : } \\
\text { MH, taches } \\
\text { blanches }\end{array}$ & $\begin{array}{l}\text { MH, taches } \\
\text { blanches }\end{array}$ & AR \\
\hline$?$ & $?$ & $20 q 13.2$ & ET3 & 2 & $\begin{array}{c}\text { Lethal spotting (Is) } \\
\mathrm{MH} \text {, taches } \\
\text { blanches }\end{array}$ & $\begin{array}{c}\text { MH, taches } \\
\text { blanches }\end{array}$ & AR \\
\hline$?$ & $?$ & $6 p 23-24$ & ET1 & & & $\begin{array}{c}\text { Malformations } \\
\text { crânio-faciales, } \\
\text { HTA }\end{array}$ & AR \\
\hline$?$ & $?$ & $1 p 34$ & ET2 & & & - & \\
\hline Sp & MH et trisomie 21 & $21 q$ & $?$ & & & - & \\
\hline Sp & $\begin{array}{c}\text { MH et délétion } \\
13 q 24\end{array}$ & $13 q 24$ & $?$ & & & - & \\
\hline$?$ & $?$ & $22 q$ & $?$ & 15 & $\begin{array}{c}\text { Dominant spotting } \\
\text { (dom): } \\
\text { MH, taches } \\
\text { blanches }\end{array}$ & & $A D$ \\
\hline
\end{tabular}

Principaux gènes, loci et anomalies chromosomiques humains et murins (italiques) associés à la maladie de Hirschsprung (MH) et à des phénotypes apparentés: endocrinopathies néoplasiques multiples (MEN 2A, MEN 2B), cancer médullaire familial de la thyroïde (FMTC), anomalies pigmentaires. $A D$ : autosomique dominant; $A R$ : autosomique récessif; $S p$ : sporadique; ET: endothélines; EDNRB : gène codant pour le récepteur $\beta$ des endothélines; RET: proto-oncogène récepteur tyrosine-kinase.

[17] : rétinites pigmentaires (mutations de la rhodopsine), certaines précocités pubertaires familiales masculines (mutations du récepteur de la LH), hypercalciuries hypercalcémiques familiales (récepteurs sensibles au calcium), adénomes thyroïdiens (récepteur de la TSH), diabètes insipides néphrogéniques liés à l'X (récepteur de la vasopressine 2), ou encore, déficits familiaux en glucocorticoïde (récepteur de l'ACTH). Il est remarquable qu'aucune de ces mutations ne touche une région homologue du cinquième domaine transmembranaire de EDNRB.
Bien que la mutation $\mathrm{W}^{276} \rightarrow \mathrm{C}$ soit absente chez 90 individus témoins (180 chromosomes), il a fallu étayer sa responsabilité dans le phénotype observé par l'étude de son effet in vitro dans des cellules $\mathrm{CHO}$, transfectées par des versions normales ou mutantes de l'ADNc EDNRB. Sous l'action d'un agoniste sélectif de ce récepteur, la saraphotoxine C, l'élévation du calcium intracellulaire enregistrée est très significativement inférieure dans le cas de l'ADNc mutant $\mathrm{W}^{276} \rightarrow \mathrm{C}$ (d'un facteur 3 à 4). Cependant, cette mutation ponctuelle a un comportement génétique inhabituel. Alors qu'un tel effet fondateur couplé à la consangui- nité familiale concorde avec un modèle autosomique récessif, seuls $60 \%$ des filles et $85 \%$ des garçons homozygotes $\mathrm{W}^{276} \rightarrow \mathrm{C} / \mathrm{W}^{276} \rightarrow \mathrm{C}$ ont une $\mathrm{MH}$ et des sujets hétérozygotes pour la mutation sont également atteints $(21 \%)$ : cette mutation n'est donc ni complètement dominante, ni complètement récessive [14] ! De façon encore plus surprenante, $9 \%$ à $13 \%$ des individus homozygotes normaux sont atteints de $\mathrm{MH}$ : cette mutation ne suffit donc pas à expliquer le phénotype dans cette famille. Enfin, son effet dépendant du sexe indique encore clairement l'existence d'autres gènes modificateurs de son expression. 
Cette découverte pourrait donc ne pas totalement convaincre si Hosada et al. ne rapportaient pas dans le même numéro de Cell l'invalidation ciblée du gène $E D N R B$ de souris par recombinaison homologue qui conduit à un mégacôlon aganglionique avec anomalies de pigmentation de pelage [18]. Ces auteurs démontrent aussi par croisement des deux souches que la mutation $E D N R B$ est allélique des mutants spontanés piebald lethal (ls) et piebald (s) dont le locus était déjà cartographié sur le chromosome 14 de la souris, synténique du chromosome $13 q$ humain. Le phénotype piebald lethal est dû à une délétion complète du gène $E D N R B$ (pas d'ARNm), alors que le phénotype piebald, moins sévère (défaut de pigmentation uniquement), est probablement dû à une mutation ponctuelle du gène conduisant à une diminution de son transcrit $(28 \%$ de l'abondance de l'ARNm en Northern blot). Ces données sont particulièrement intéressantes puiqu'elles achèvent de démontrer l'hétérogénéité génétique du mégacôlon congénital s'il en était besoin (Tableau I). Cependant, de nombreuses questions restent posées parmi lesquelles:

- Quelle est la proportion de patients atteints de MH et qui présentent une mutation du gène EDNRB? Si l'on en croit la rareté des anomalies pigmentaires chez ces malades et les exceptionnels pedigrees récessifs dans les familles de mégacôlon, le rôle de ce gène pourrait être quantitativement peu important. Cela doit conduire à une analyse moléculaire fine du gène $E D N R B$, dans les cas sporadiques et familiaux de $\mathrm{MH}$, ce d'autant qu'ils ont fait la preuve de leur exclusion du locus RET.

\footnotetext{
- Quelles indications physiologiques peut-on tirer de cette information et, en particulier, quel rôle normal peut être assigné aux endothélines et à leurs récepteurs dans le développement des cellules originaires des crêtes neurales, neurones entériques et mélanocytes de la peau? Les endothélines sont une famille de peptides de 21 acides aminés qui provoquent
}

longue durée. Ces trois peptides sont codés par des gènes différents et l'on connaît à ce jour plusieurs types de récepteurs dont l'expression tissulaire est variable et spécifique de l'un ou l'autre des ligands. Les endothélines exercent aussi des effets non vasculaires sur le cour, le rein, le cerveau et le foie où elles se conduisent en neuropeptides et, même, en facteurs de croissance. Or, il est postulé que la $\mathrm{MH}$ est un défaut de développement des cellules des crêtes neurales touchant leur migration, leur différenciation ou leur colonisation de l'intestin. Ce phénotype pourrait être lié à des anomalies des interactions cellules/cellules ou cellules/ substrat au cours du développement des neuroblastes primitifs ou du microenvironnement intestinal. Il est donc clair que la mutation d'un récepteur des endothélines dans la $\mathrm{MH}$ pose la question d'une fonction nouvelle de ces molécules dans le contrôle du développement des neurones entériques. A moins que l'hypothèse vasculaire à l'origine de la MH ne trouve ici des arguments, le rôle des endothélines dans la trophicité vasculaire et dans la régulation de la pression artérielle pouvant laisser penser que les mutations du récepteur béta conduisent à des anomalies du microenvironnement vasculaire intestinal et à une ischémie rendant peu favorable l'implantation des neuroblastes originaires des crêtes neurales. Mais alors, comment expliquer les anomalies des mélanocytes?

- Laquelle des trois endothélines est le ligand physiologique de EDNRB en ce qui concerne le développement des cellules des crêtes neurales, des mélanocytes, des neurones entériques? Dans un troisième article de la même livraison de Cell, les mêmes groupes de Dallas et de Montréal montrent que des souris mutantes pour le gène codant pour l'endothéline 3 présentent un phénotype identique: mégacôlon avec taches blanches [19]. Cette mutation est allélique de lethal spotting, dont la mutation a été identifiée: il s'agit d'une substitution arginine $\rightarrow$ tryptophane abolissant le site de clivage protéolytique de la pré-proendothéli- ne en endothéline mûre. Cela permet d'établir que l'endothéline 3 est vraisembablement le ligand naturel de EDNRB en ce qui concerne les cellules des crêtes neurales. Si l'on se souvient du rôle de l'endothéline 1 (ET 1) dans le développement crâniofacial $\left(m / s n^{\circ} 6-7\right.$, vol. 10, p. 740) [20], on est vraiment conduit à envisager des fonctions nouvelles des récepteurs des endothélines et de leurs ligands dans le développement, ce qui constitue, à n'en pas douter, une surprise mais aussi une formidable piste de recherche dans le domaine de l'embryologie moléculaire. A quand le troisième gène de la $\mathrm{MH}$ qui nous permettrait encore, grâce à l'étude des patients, de démasquer de nouveaux acteurs du mystérieux développement du système nerveux entérique et des cellules des crêtes neurales?

T.A.

J.A.

P.E.

S.L.

1. Hirschsprung H. Stuhltragheit neugeborener infolge von dilatation und hypertrophic des colons. Jo Kinderheilk 1887; 27 : 1 .

2. Bolande RP. The neurocristopathies; a unifying concept of disease arising in neural crest maldevelopment. Hum Pathol 1973; 5 : 409-29.

3. Badner JA, Sieber WK, Garver KL, Chakravarti A. A genetic study of Hirschsprung disease. $\mathrm{Am}$ J Hun Genet 199(); 46: 568-80).

4. Lyonnet S, Bolino A, Pelet A, Abel L, NihoulFékété C: Briard ML, MokSiu V, Kaariainen $\mathrm{H}$ Martucciello G, Lerone M, Puliti A, Luo Y, W'eissenbach J, Devoto M, Munnich A, Romeo (;. A gene for Hirschsprung disease maps to the proximal long arm of chromosome 10. Nature Genet $1993 ; 4: 346-50$.

5. Romeo G, Ronchetto P, Luo Y, Barone V, Seri M, Ceccherini l, Pasini B, Bocciardi R, Lerone M, Kaariainen H, Martucciello (;. Point mutations affecting the tyrosine kinase domain of the RET proto-oncogene in Hirschsprung's disease. Nature $1994 ; 367: 377-8$

6. Edery P, Lyonnet S, Mulligan LM, Pelet A, Dow E, Abel L, Holder S, Nihoul-Fékété C, Ponder BAl, Munnich A. Mutations of the RET protooncogene in Hirschsprung's disease. Nature 1994; $367: 378-80$.

7. Lyonnet S, Edery P, Nihoul-Fékété C, Munnich A. Un gène de la maladie de Hirschsprung localise sur le bras long du chromosome 10: une brèche dans le modèle multifactoriel des malformations de l'enfant. médecine/sriences 1993; 9: 994-5. 
8. Lyonnet S, Edery P, Attié T, Nihoul-Fékété C, Munnich A. Des mutations du proto-oncogène RET dans la maladie de Hirschsprung: un gène à tout faire! medecine/sciences $1994 ; 10: 450$ )-3.

9. Pelet A, Attié T, Goulet O, Eng C, Ponder BAJ, Munnich A, Lyonnet S. De nozo mutations of the RET proto-oncogene in Hirschsprung's disease. Lancet $1994 ; 344: 1769-70$.

10. Luo Y, Barone V, Seri M, Bolino A, Bocciardi $R$, Ceccherini 1 , Pasini $B$, Tocco $T$, Lerone $M$, Ciwes S, Moore S, Vandenwinden JM, Abramowicz M], Kristoffersson U, Larsson LT, Hamel BC.J, Silengo M, Martucciello (;, Romeo (;. Heterogeneity and low detection rate of RET mutations in Hirschsprung disease. Eur J Hum Genet 1994; 2 : 272-80

11. Attié T, Pelet A, Edery P, Eng C, Mulligan I.M Amiel J, Boutran L, Beljord C, Munnich M, Lyonnet $S$. Diversity of RET mutations in Hirschsprung disease. Hum Mol (ipnet 1995 (sous presse).

12. Attié T, Till M, Pelet A, Edery P, Munnich A, Lyonnet S. Exclusion of the RET and Pax3 loci in W'aardenburg-Hirschsprung's disease. I Med ('enet $1995 ; 32: 312-3$.

13. Puffenberger EG, Kauffman ER, Bolk S, Matise TC, W'ashington SS, Angrist M, W'eissenbach J, Garver KL, Mascari M, Ladda R, Slaugenhaupt SA, Chakravarti A. Identity-by-descent and association mapping of a recessive gene for Hirschsprung disease on human chromosome $13 q 22$. Hum Mol Genet 1994; 3: 1217-25.

14. Puffenberger EG, Hosoda K, Washington SS Nakao K, deWit D, Yanagisawa N, Chakravarti A. A missense mutation of the endothelin- $B$ receptor gene in multigenic Hirschsprung's disease. Cell $1994 ; 79$ : 1257-66.

15. Lotersztajn S. Les endothélines. médecine/scienres $1993 ; 9$ : 1084-93.

16. Hibert MF, Hoflack J, Trumpp-Kallmeyer $S$ Bruinvels A. Modèles tridimensionnels des récep teurs couplés aux protéines (;. médecine/sciences $1993 ; 9$ : 31-40

17. Bockaert J. Les récepteurs à sept domaines transmembranaires: physiologie et pathologie de la transduction. médecine/sciences $1995 ; 11: 382-94$.

18. Hosada K, Hammer RE, Richardson JA, Baynash AC, Cheung JC, Giaid A, Yanagisawa M. Targeted and natural (Piebald-Lethal) mutations of endothelin-B receptor gene produce megacolon associated with spotted coat color mice. Cell 1994; 79: 1267-76.

19. Baynash AG, Hosada K, Giaid A, Richardson RE, Emoto N. Hammer RE, Yanagisawa M. Interaction of endothelin- 3 with endothelin- $B$ receptor is essential for development of epidermal melanocytes and enteric neurons. Cell 1994; 79: 1277-85.

20. Kurihara Y, Kurihara H, Suzuki H, Kodama T, Maemura K, Nagai R, Oda H, Kuwaki T, Cao WH, Kimada N, Jishage K, Ouchi Y, Azuma S, Toyoda Y, Ishikawa T, Kumada M, Yazaki Y. Elevated blood pressure and craniofacial abnormalities in mice deficient in endothelin-1. Nature 1994; 368 : 703-10.

\section{BRÈVES}

AMP cyclique et mémoire (suite). Chez la drosophile, plusieurs mutations affectant la mémoire avaient dé jà été reliées à des gènes codant pour des enzymes de la synthèse ou de la dégradation de l'AMPc: le gène dunce, le premier identifié, code pour une phosphodiestérase; le gène rutabaga, pour une adénylyl-cyclase $\left(\mathrm{m} / \mathrm{s} n^{\circ} 5\right.$, vol. 8 , p. 491). Par la suite, d'autres mutations ont été caractérisées, parmi lesquelles amnesiac. Le crible utilisé pour détecter ces mutations consiste à associer une odeur à un choc électrique et à déterminer celles des mouches qui oublient rapidement cette association, et par conséquent n'ont plus tendance à éviter l'odeur conditionnante. Feany et Quinn, de Boston et Cambridge (MA, USA), ont utilisé la méthode de mutagenèse par insertion d'éléments $P$, ce qui permet aisément de repérer le gène inactivé par mutagenèse insertionnelle [1]. Un des mutants ainsi isolé avait un phénotype tout à fait similaire à amnesiac. Le gène correspondant code pour un peptide ressemblant à l'activateur pituitaire de l'adénylyl-cyclase, le PACAP (pituita$r y$ adenylyl cyclase activating peptide). Le rôle de l'AMP cyclique dans les phénomènes de mémoire a également été confirmé chez la drosophile en provoquant l'expression conditionnelle d'un inhibiteur de la protéine CREB, un activateur transcriptionnel activé par la protéine kinase A sous l'effet de l'AMPc [2]. Il est donc probable que, au moins chez la drosophile, la mémorisation soit associée à des phénomènes de phosphorylation dépendant de la protéine kinase A. Des neuro-transmetteurs pourraient aboutir à un flux entrant de calcium qui activerait l'adénylyl cyclase Rutabaga, la teneur en AMPc étant contrôlée par l'action de la phosphodiestérase Dunce. Cette réaction pourrait être elle-même modulée par des terminaisons peptidergiques libérant des peptides de type PACAP. Il faut noter que, chez les vertébrés, les modifications post-traductionnelles associées aux phénomènes de mémorisation pourraient être beaucoup plus compliquées que chez la drosophile et mettre en jeu de nombreux autres systèmes de protéine kinases et protéine phosphatases que ceux dépendant de l'AMPc. [1. Feany MB, Quinn WG. Science $1995 ; 268$ : 869-73.]

[2. Yin JCP, et al. Cell 1995; 81 : 10716.]

Des plantes transgéniques utilisées comme vaccin oral. Nous avons vu, dans un tout récent numéro de médecine/sciences, que des plantes transgéniques synthétisant selon différents procédés des protéines vaccinales pourraient constituer, dans l'avenir, une source commode et bon marché de vaccins d'un nouveau type $\left(m / s n^{\circ} 6\right.$, vol. 11 , p. 926). Cependant, la purification totale des protéines recombinantes à partir des extraits de plantes transgéniques reste complexe. Hak et al., de Houston (TX, USA), montrent que, dans certains cas, l'ingestion de plantes transgéniques pourrait conduire simplement au développement d'une immunité muqueuse [1]. Des plants de tabac transgénique sont construits, qui synthétisent l'entérotoxine sensible à la chaleur d'Escherichia coli. L'ingestion par des souris de la plante ellemême permet d'obtenir une immunisation, avec augmentation des anticorps spécifiques dans le sérum et dans les sécrétions muqueuses. Le même résultat est obtenu si l'antigène est synthétisé par des pommes de terre. Les auteurs ont également étendu ces résultats à un autre antigène, la protéine de capside du virus de Norwalq. Quoique cette vision reste futuriste, on peut déjà imaginer la culture de plantes vaccinant leur consommateur contre une série de pathogènes, notamment en milieu tropical.

[1. Haq TA, et al. Science 1995; 268 : 414-6.]

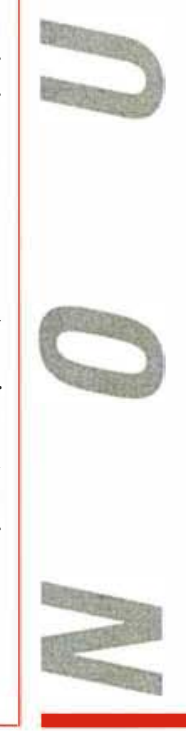

